\title{
Volatilização de amônia e emissão de óxido nitroso após aplicação de dejetos líquidos de suínos em solo cultivado com milho
}

\author{
Ammonia volatilization and nitrous oxide emissions following soil application \\ of pig slurry in corn
}

\author{
Rogério Gonzatto $^{I^{*}}$ Ezequiel Cesar Carvalho Miola ${ }^{\mathrm{I}}$ Alexandre Doneda ${ }^{\mathrm{I}}$ Stefen Barbosa Pujol ${ }^{\mathrm{I}}$ \\ Celso Aita ${ }^{\text {II }}$ Sandro José Giacomini ${ }^{I I}$
}

RESUMO

As perdas de nitrogênio ( $N$ ) através de emissões gasosas de amônia $\left(\mathrm{NH}_{3}\right)$ e óxido nitroso $\left(\mathrm{N}_{2} \mathrm{O}\right)$ após a aplicação de dejetos líquidos de suínos (DLS) no solo é um assunto ainda pouco estudado no Brasil, sobretudo em plantio direto. Diante disso, o objetivo desse trabalho foi avaliar, em condições de campo, a volatilização de $\mathrm{NH}_{3}$ e a emissão de $\mathrm{N}_{2} \mathrm{O}$ após a aplicação de DLS no milho, com e sem a presença de palha de aveia preta na superfície do solo. Os tratamentos avaliados para medir a emissão de $\mathrm{N}_{2} \mathrm{O}$, durante 90 dias, foram: $\mathrm{T} 1$ - Solo (testemunha); T2 - Solo + DLS; T3 - Solo + Palha; T4 - Solo + Palha + DLS. Já a volatilização de $\mathrm{NH}_{3}$ foi quantificada durante 12 dias, somente nos três últimos tratamentos. Os DLS, na dose de $60 \mathrm{~m}^{3} \mathrm{ha}^{-1}$, foram aplicados ao solo, antecedendo a semeadura do milho, em delineamento experimental de blocos ao acaso, com três repetições. A presença da palha na superfície do solo reduziu as perdas de $\mathrm{NH}_{3}$ em 34\%, porém potencializou as emissões de $\mathrm{N}_{2} \mathrm{O}$ após a aplicação dos DLS, as quais aumentaram em $167 \%$. Os maiores fluxos de $\mathrm{N}_{2} \mathrm{O}$ ocorreram durante os primeiros 40 dias após a aplicação dos DLS sempre após a ocorrência de chuvas e/ ou irrigações.

Palavras-chave: emissão de gases de efeito estufa, perdas de $\mathrm{N}$, palha, efluente de suinocultura.

\section{ABSTRACT}

The nitrogen $(N)$ losses through gaseous emissions of ammonia $\left(\mathrm{NH}_{3}\right)$ and nitrous oxide $\left(\mathrm{N}_{2} \mathrm{O}\right)$ after pig slurry (PS) application in soil is a subject still little studied in Brazil, especially under no-till conditions. Therefore, the objective of this study was to evaluate, under field conditions, the $\mathrm{NH}_{3}$ volatilization and the $\mathrm{N}_{2} \mathrm{O}$ emission after PS application in corn, with and without surface oat straw. The treatments for measuring $\mathrm{N}_{2} \mathrm{O}$ emissions, during 90 days, were: T1-unamended soil (control), T2 - Soil + PS, T3 - Soil + oat straw and T4 - Soil + PS + oat straw. The
$\mathrm{NH}_{3}$ volatilization was quantified only in the last three treatments. $P S$, at the dose $60 \mathrm{~m}^{3} \mathrm{ha}^{-1}$, was applied prior the sowing of corn, in a randomized design, with three replications. Oat straw on the soil surface reduced $\mathrm{NH}_{3}$ volatilization by $34 \%$, but enhanced $\mathrm{N}_{2} \mathrm{O}$ emission by $167 \%$. The higher $\mathrm{N}_{2} \mathrm{O}$ fluxes occurred during the first 40 days after PS addition, always after rain and/or irrigation events.

Key words: greenhouses-gas emissions, $N$ losses, straw, pig farm effluent.

\section{INTRODUÇÃO}

A suinocultura é uma atividade de grande importância econômica e social para o setor agropecuário brasileiro. Dados recentes mostram o Brasil como o quarto maior produtor e exportador de carne suína do mundo (ABIPECS, 2012). Essa atividade suinícola está concentrada, principalmente, em regiões declivosas (KUNZ et al., 2009) dos estados da Região Sul e, sobretudo, caracteriza-se pela geração de grande volume de dejetos, os quais são manejados na forma líquida e são ricos em $\mathrm{N}$ amoniacal (MEIJIDE et al., 2007). Devido ao seu elevado conteúdo em $\mathrm{N}$, os DLS são aplicados no solo como uma alternativa de adubação nitrogenada às culturas e como uma forma de descarte desses resíduos. No entanto, a carga poluente dos DLS pode afetar negativamente outros ambientes como os corpos d'água, propiciando o crescimento de microorganismos e a ocorrência de eutrofização (MEADE et al., 2011).

IPrograma de Pós-graduação em Ciência do Solo, Universidade Federal de Santa Maria (UFSM), Avenida Roraima, 1000, 97105-900,

Santa Maria, RS, Brasil. E-mail: rogonzatto@gmail.com.br.*Autor para correspondência.

"Departamento de Solos, UFSM, Santa Maria, RS, Brasil. 
A aplicação dos DLS na superfície do solo contribui também para aumentar as perdas de $\mathrm{N}$ para atmosfera, principalmente nas formas gasosas de $\mathrm{NH}_{3}$ e $\mathrm{N}_{2} \mathrm{O}$, o que reduz o potencial fertilizante dos dejetos e impacta negativamente o ambiente. Para reduzir tais perdas, é importante identificar a intensidade com que elas ocorrem e também, como variam em função do método, a época e a taxa de aplicação, da composição dos dejetos, do tipo do solo e das condições ambientais (HOEKSTRA et al., 2010). Embora as emissões de $\mathrm{NH}_{3}$ e de $\mathrm{N}_{2} \mathrm{O}$ já tenham sido avaliadas após a aplicação dos DLS no Sul do Brasil (BASSO et al., 2004; GIACOMINI et al., 2006), o efeito da aplicação dos DLS na presença ou na ausência de resíduos culturais na superfície do solo sobre tais emissões ainda não é bem conhecido.

Segundo PETERSEN \& SOMMER (2011), a adição de dejetos no solo pode resultar em efeitos antagônicos sobre as emissões de $\mathrm{NH}_{3}$ e $\mathrm{N}_{2} \mathrm{O}$. Prevalecendo as formas amoniacais de $\mathrm{N}$, podem ser favorecidas as perdas de $\mathrm{N}$ como $\mathrm{NH}_{3}$, logo são reduzidas as emissões de $\mathrm{N}_{2} \mathrm{O}$. Por outro lado, a adoção de medidas que reduzam as perdas de $\mathrm{N}$ por volatilização de $\mathrm{NH}_{3}$, diminuem a disponibilidade dessa forma de $\mathrm{N}$ às bactérias nitrificadoras, reduzindo, assim, as emissões de $\mathrm{N}_{2} \mathrm{O}$, que podem ocorrer tanto durante os processos microbianos de nitrificação como de desnitrificação (PETERSEN \& SOMER, 2011). Além do N, a disponibilidade de carbono (C), que é aumentada pela adição dos DLS e pela presença da palha, pode influenciar na emissão desses gases, já que as bactérias heterotróficas, responsáveis pela imobilização de $\mathrm{N}$ e pela desnitrificação, dependem de fontes de $\mathrm{C}$ e energia. Por isso, a avaliação de como os DLS aplicados ao solo interferem na magnitude das emissões de $\mathrm{NH}_{3}$ e $\mathrm{N}_{2} \mathrm{O}$ para atmosfera é fundamental para o desenvolvimento de estratégias de manejos mais adequadas para esse material orgânico.

Com base no exposto, o objetivo do presente trabalho foi avaliar as perdas de $\mathrm{N}$ para a atmosfera por volatilização de $\mathrm{NH}_{3}$ e emissão de $\mathrm{N}_{2} \mathrm{O}$ após a aplicação de DLS no milho, com e sem a presença de resíduos culturais de aveia na superfície do solo.

\section{MATERIAL E MÉTODOS}

O experimento foi realizado em condições de campo, na área experimental do setor de Irrigação e Drenagem da Universidade Federal de Santa Maria (UFSM), RS, localizada a $29^{\circ} 41^{\prime} 24^{\prime \prime} \mathrm{S}, 53^{\circ} 48^{\prime} 42^{\prime \prime}$ W e altitude de $106 \mathrm{~m}$. O clima, segundo a classificação de Köppen, é subtropical úmido, tipo $\mathrm{Cfa} 2$. O solo é classificado como Argissolo Vermelho Distrófico arênico (Hapludalf) (SANTOS, 2006). As emissões de $\mathrm{NH}_{3}$ e as emissões de $\mathrm{N}_{2} \mathrm{O}$ foram avaliadas, de janeiro a fevereiro de 2011 (durante 13 dias) e de janeiro a abril do mesmo ano (durante 90 dias).

$\mathrm{O}$ delineamento experimental utilizado foi o de blocos ao acaso, com três repetições, em parcelas de $1,6 \mathrm{~m}^{2}(1 \mathrm{mx} 1,6 \mathrm{~m})$. Os tratamentos para avaliação da emissão de $\mathrm{N}_{2} \mathrm{O}$ consistiram da aplicação, ou não, de DLS na superfície do solo, com ou sem palha de aveia: T1 - Solo (Testemunha); T2 - Solo + DLS (DLS); T3 - Solo + Palha de aveia (Palha); T4 - Solo + Palha + DLS (Palha + DLS). Enquanto para análise da volatilização de $\mathrm{NH}_{3}$ foram utilizados somente os tratamentos "Palha", "DLS" e "Palha + DLS", os DLS usados nesses dois experimentos foram obtidos em uma esterqueira anaeróbica no setor de suinocultura da UFSM, a partir da dejeção de animais em regime de terminação. A aplicação dos dejetos foi realizada manualmente, com regadores, em 19/01/2011, na dose de $60 \mathrm{~m}^{3} \mathrm{ha}^{-1}$, antecedendo em dois dias a semeadura do milho (híbrido Pioneer P3646H). Nos dejetos, foram analisados o $\mathrm{pH}$, os teores de matéria seca (MS), de $\mathrm{N}$ total e amoniacal (TEDESCO et al., 1995).

No tratamento Palha + DLS, os dejetos foram adicionados ao solo sobre 4,0 $\mathrm{Mg} \mathrm{ha}^{-1}$ de palha de aveia preta (Avena strigosa Schreb), a qual foi coletada em área próxima, no estágio de maturação fisiológica. A matéria seca da palha foi obtida secando-se o material em estufa a $65^{\circ} \mathrm{C}$, e os teores de $\mathrm{C}$ orgânico e $\mathrm{N}$ total, foram determinados conforme método descrito por TEDESCO et al. (1995). As quantidades de palha, $\mathrm{C}$ e $\mathrm{N}$, além do $\mathrm{pH}$ dos dejetos são mostrados na tabela 1 .

Os fluxos de $\mathrm{N}_{2} \mathrm{O}$ foram medidos usando o método da câmara fechada, seguindo os procedimentos recomendados por ROCHETTE \& BERTRAND (2008). O sistema de coleta foi composto por uma base e por uma câmara, ambas em aço galvanizado. A cada coleta, as câmaras $(0,3 \mathrm{~m}$ de largura $\mathrm{x} 0,2 \mathrm{~m}$ de altura $\mathrm{x} 0,4 \mathrm{~m}$ de comprimento) foram dispostas sobre as bases $(0,3 \mathrm{~m}$ de largura $\mathrm{x} 0,04 \mathrm{~m}$ de altura $\mathrm{x}$ $0,4 \mathrm{~m}$ de comprimento), as quais foram inseridas no solo até uma profundidade de $0,1 \mathrm{~m}$. As coletas foram realizadas de três a quatro vezes por semana durante o primeiro mês, sendo posteriormente espaçadas (uma a duas vezes a cada 15 dias), devido à similaridade das emissões aos valores obtidos no tratamento testemunha. As amostras de ar foram coletadas com seringas de polipropileno de $20 \mathrm{~cm}^{3}(0,15,30$ e 45 minutos) após o fechamento da câmara.

A concentração de $\mathrm{N}_{2} \mathrm{O}$ foi determinada por cromatografia gasosa, em cromatógrafo 
Tabela 1 - Caracterização dos dejetos líquidos de suínos (DLS) e da palha de aveia utilizados no experimento.

\begin{tabular}{|c|c|c|c|c|c|c|c|c|}
\hline \multirow{3}{*}{ Resíduo Orgânico } & \multirow{3}{*}{ MS } & \multirow{3}{*}{ MS } & \multirow{3}{*}{$\mathrm{C}$} & ------- & ----N------- & --------------' & \multirow{3}{*}{$\mathrm{C} / \mathrm{N}$} & \multirow{3}{*}{$\mathrm{pH}$} \\
\hline & & & & & & & & \\
\hline & & & & Total & Amoniacal & Orgânico & & \\
\hline & $\%$ & 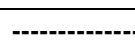 & - & $--\mathrm{kg}$ ha & |----------- & ------------- & & \\
\hline $\operatorname{DLS}^{(1)}$ & 1,4 & 890 & 328 & 160 & 119 & 41 & 2,1 & 8,1 \\
\hline Palha $^{(2)}$ & - & 4.000 & 1.560 & 62,3 & - & - & 25,0 & - \\
\hline
\end{tabular}

Em que: ${ }^{(1)}$ Em base úmida; ${ }^{(2)}$ Em base seca; MS: matéria seca; C: carbono; N: Nitrogênio.

(Shimadzu, modelo GC-2014 Greenhouse) equipado com coluna empacotada $\left(80^{\circ} \mathrm{C}\right)$, com detector de ionização em chamas Flame Ionization Detector (FID) para determinação de metano $\left(\mathrm{CH}_{4}\right)$, com metanador, o qual transforma o $\mathrm{CO}_{2}$ em $\mathrm{CH}_{4}$, para a determinação de dióxido de carbono, e de detector de captura de elétrons Electron Capture Detector (ECD) para a quantificação de $\mathrm{N}_{2} \mathrm{O}$. Os fluxos de $\mathrm{N}_{2} \mathrm{O}$ foram calculados utilizando a equação proposta por ROCHETTE \& BERTRAND (2008):

$$
F g=\frac{d G}{d t} \times \frac{V}{A} \times \frac{M m}{V m} x\left(1-\frac{e p}{P}\right)
$$

em que $\mathrm{F}_{\mathrm{g}}$ é o fluxo de gás $\left(\mathrm{g} \mathrm{m}^{-2} \mathrm{~s}^{-1}\right), \mathrm{dG} / \mathrm{dt}$ é a mudança na concentração do gás $\left(\mathrm{mol} \mathrm{mol}^{-1} \mathrm{~s}^{-1}\right), \mathrm{V}$ é o volume da câmara $\left(\mathrm{m}^{3}\right)$, A é a área coberta pela câmara $\left(\mathrm{m}^{2}\right)$, ep é a pressão parcial do vapor de água no interior da câmara $(\mathrm{kPa}), \mathrm{P}$ é a pressão barométrica $(\mathrm{kPa}), \mathrm{M}_{\mathrm{m}}$ é a massa molecular do gás $\left(\mathrm{g} \mathrm{mol}^{-1}\right), \mathrm{V}_{\mathrm{m}}$ é o volume molecular sob condições de temperatura e pressão barométrica internas à câmara $\left(\mathrm{m}^{3} \mathrm{~mol}^{-1}\right)$.

As avaliações de $\mathrm{NH}_{3}$ foram realizadas utilizando câmaras estáticas semi-abertas, conforme proposto por NÖMMIK (1973), com algumas modificações. As câmaras foram construídas a partir de chapas de cloro-polivinil (PVC), com 0,3m de lado e $0,4 \mathrm{~m}$ de altura, dispostas sobre uma base metálica com $0,04 \mathrm{~m}$ de altura, as quais foram inseridas no solo até uma profundidade de $0,1 \mathrm{~m}$.

Para captar a amônia volatilizada, foram utilizadas espumas de densidade 28, com $0,02 \mathrm{~m}$ de espessura do mesmo formato da câmara. Cada câmara era dotada de duas espumas, sendo uma situada a $0,15 \mathrm{~m}$ da superfície do solo, para captar a $\mathrm{NH}_{3}$ emitida do solo e outra a $0,15 \mathrm{~cm}$ acima da espuma anterior para impedir a entrada de $\mathrm{NH}_{3}$ externa à câmara. As espumas foram embebidas com $100 \mathrm{~mL}$ de uma solução preparada com $50 \mathrm{~mL}$ de ácido fosfórico $\left(\mathrm{H}_{3} \mathrm{PO}_{4}-85 \% \mathrm{PA}\right)$ e $40 \mathrm{~mL} \mathrm{~L}^{-1}$ de glicerina. As avaliações foram realizadas após 7, 22, 54, 103, 126, 150, 191, 258, 279, 303 horas da aplicação dos dejetos. A cada coleta, a esponja inferior foi trocada e a superior mantida até o final do período de avaliação.
As espumas trocadas foram lavadas com solução de $\mathrm{KCl}\left(1,0 \mathrm{~mol} \mathrm{~L}^{-1}\right)$ até completar um volume de $1000 \mathrm{~mL}$ de solução. Desse volume, foi retirada uma alíquota de $20 \mathrm{~mL}$ para determinação do teor de $\mathrm{N}-\mathrm{NH}_{3}$ em destilador semi-micro Kjeldahl, conforme TEDESCO et al. (1995).

Os resultados foram submetidos à análise de variância e as médias dos tratamentos comparadas entre si pelo teste de Tukey a $5 \%$ de probabilidade de erro.

\section{RESULTADOS E DISCUSSÃO}

Emissão de $\mathrm{N}_{2} \mathrm{O}$

As emissões de $\mathrm{N}_{2} \mathrm{O}$ aumentaram logo após a aplicação dos dejetos, principalmente quando eles foram aplicados sobre os resíduos culturais de aveia (Palha + DLS) (Figura 1a). Na primeira avaliação, realizada 25 horas após a aplicação dos DLS, ocorreu um aumento nos fluxos de $\mathrm{N}_{2} \mathrm{O} 226,27 \mu \mathrm{g}$ de $\mathrm{N}_{-} \mathrm{N}_{2} \mathrm{O}$ $\mathrm{m}^{-2} \mathrm{~h}^{-1}$ no tratamento Palha + DLS em relação ao tratamento sem dejetos (Palha). Comportamento semelhante foi observado por PERALA et al. (2006), no Sul da Finlândia, quando aplicaram dejetos de suínos sobre a palha de cevada. Em condições semelhantes às do presente trabalho, GIACOMINI et al. (2006) verificaram que a aplicação de $40 \mathrm{~m}^{3}$ ha $^{-1}$ de DLS (154kg ha-1 de $\mathrm{N}$ total), juntamente com resíduos culturais de aveia preta $\left(3,6 \mathrm{Mg} \mathrm{ha}^{-1}\right)$ aumentou as emissões de $\mathrm{N}_{2} \mathrm{O}$ em relação ao solo sem dejetos, principalmente nos primeiros 15 dias, tanto no plantio direto, como no preparo reduzido do solo. Segundo os autores, as maiores emissões de $\mathrm{N}_{2} \mathrm{O}$, logo após a aplicação dos dejetos, e que também podem explicar os resultados do presente trabalho, podem ser atribuídas, sobretudo, a três fatores: 1) os dejetos apresentam fração do $\mathrm{C}$ prontamente disponível aos microrganismos, o que proporciona aumento na atividade microbiana, resultando em elevada demanda de $\mathrm{O}_{2}$. Isso pode gerar sítios de anaerobiose, favorecendo a emissão de $\mathrm{N}_{2} \mathrm{O}$, a partir da utilização do $\mathrm{NO}_{3}^{-}$presente no solo, por bactérias anaeróbias 


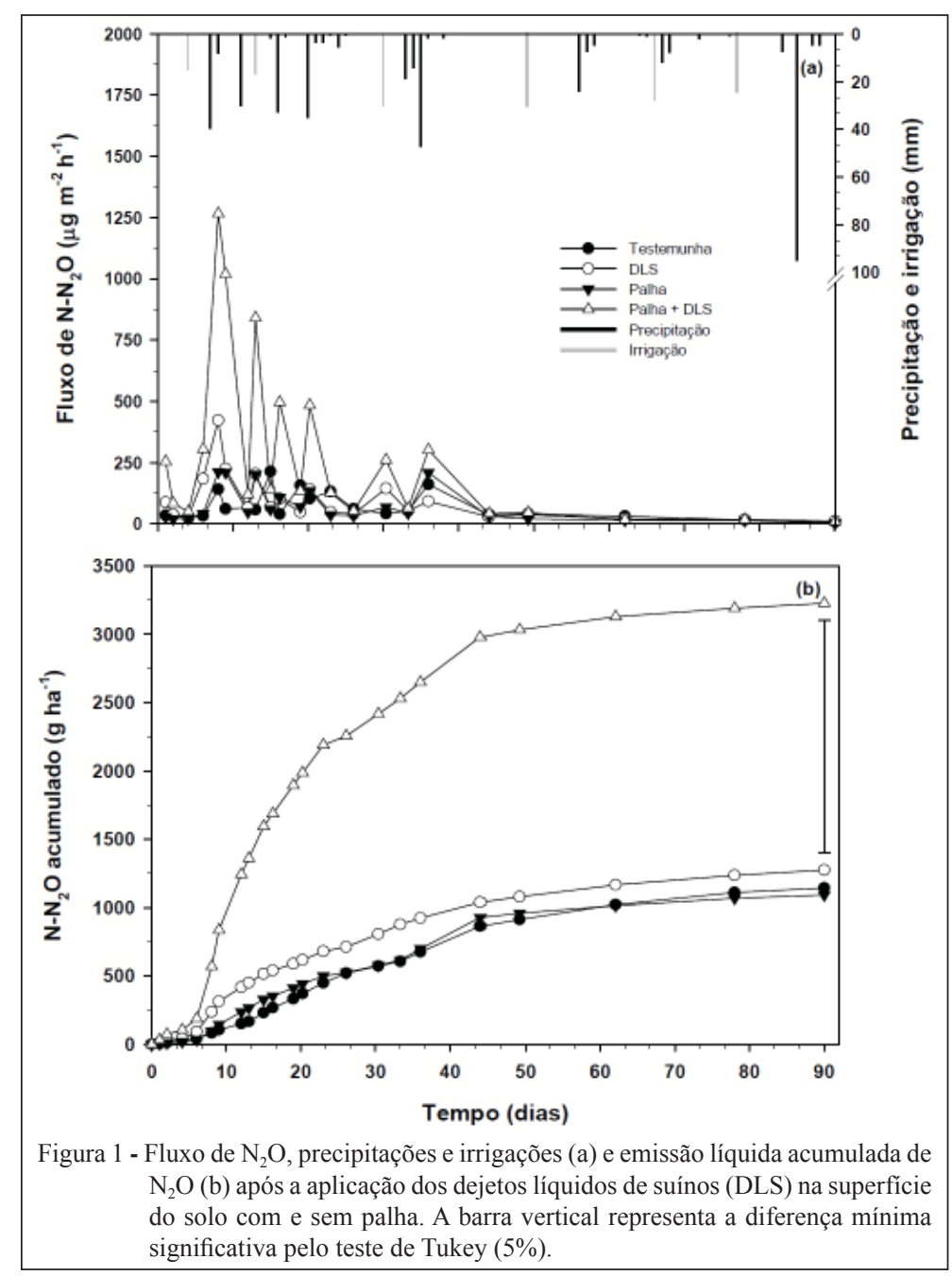

facultativas, capazes de substituir o $\mathrm{O}_{2}$ pelo $\mathrm{NO}_{3}$ como aceptor final de elétrons; 2) durante o processo de nitrificação do $\mathrm{NH}_{4}^{+}$aplicado com os dejetos de suínos, ocorre produção de $\mathrm{N}_{2} \mathrm{O}$, aumentando a emissão desse gás; 3 ) a alta concentração de líquidos nos dejetos de suínos pode promover a ocorrência de sítios de anaerobiose, favorecendo o processo de desnitrificação do $\mathrm{NO}_{3}{ }^{-}$no solo.

Com apenas uma irrigação de $15 \mathrm{~mm}$ e sem ocorrência de chuvas durante os primeiros quatro dias, após a aplicação dos dejetos, as emissões de $\mathrm{N}_{2} \mathrm{O}$ foram baixas e próximas ao tratamento testemunha. Todavia, a ocorrência de chuva de $40 \mathrm{~mm}$ aos sete dias potencializou a emissão de $\mathrm{N}_{2} \mathrm{O}$, principalmente no tratamento DLS + Palha (Figura 1a). Nesse tratamento, o fluxo de $\mathrm{N}_{2} \mathrm{O}$ após a chuva foi de $1266 \mu \mathrm{g}$ de $\mathrm{N}_{-} \mathrm{N}_{2} \mathrm{O} \mathrm{m} \mathrm{m}^{-2} \mathrm{~h}^{-1}$, superando o tratamento somente com a aplicação de DLS em $844 \mu \mathrm{g}$ de N-N $\mathrm{N}_{2} \mathrm{O}$ $\mathrm{m}^{-2} \mathrm{~h}^{-1}(200 \%)$. Segundo GIACOMINI et al. (2006), esse aumento das emissões de $\mathrm{N}_{2} \mathrm{O}$, normalmente observado após as primeiras precipitações, pode ser causado devido ao rompimento, pela água da chuva, da crosta formada pelas partículas orgânicas dos dejetos na superfície do solo, o que facilitaria a liberação dos gases aprisionados em zonas de anaerobiose. Além disso, a chuva pode carrear para o solo o $\mathrm{C}$ da fração solúvel dos resíduos culturais, o qual aumenta a população e a atividade da população microbiana heterotrófica do solo, incluindo as bactérias redutoras do $\mathrm{NO}_{3}{ }^{-}$. O efeito do $\mathrm{C}$ solúvel da palha no favorecimento da desnitrificação pode ser comprovado comparando-se a emissão acumulada líquida de $\mathrm{N}-\mathrm{N}_{2} \mathrm{O}$ dos dois tratamentos com aplicação de DLS. Quando os dejetos foram aplicados sobre a palha (Palha + DLS), a quantidade de $\mathrm{N}-\mathrm{N}_{2} \mathrm{O}$ aumentou em $1.951 \mathrm{~g}$ de $\mathrm{N}-\mathrm{N}_{2} \mathrm{O}$ ha $^{-1}$ (2,5 vezes) em relação ao tratamento sem palha (DLS) (Figura 1b).

Nos primeiros 36 dias após a aplicação dos dejetos, ocorreram os maiores picos nas emissões de $\mathrm{N}_{2} \mathrm{O}$, as quais estiveram relacionadas á irrigação ou 
a ocorrência de chuvas (Figura 1a). Esse período foi responsável pela emissão de aproximadamente $90 \%$ da quantidade total de $\mathrm{N}-\mathrm{N}_{2} \mathrm{O}$ emitida durante os primeiros 40 dias do experimento. A partir da décima sétima avaliação, os fluxos de $\mathrm{N}_{2} \mathrm{O}$ no tratamento com palha e nos dois tratamentos com dejetos reduziram para níveis próximos aos da testemunha. Esse resultado evidência que, a partir dos 30 dias, a atividade das bactérias desnitrificantes começou a ser limitada, principalmente pela baixa disponibilidade de $\mathrm{C}$ e de $\mathrm{NO}_{3}{ }^{-}$no solo, em razão da absorção desse ânion pelo milho.

\section{Emissão de $\mathrm{NH}_{3}$}

A aplicação de DLS no solo tanto sem a presença de resíduos culturais como sobre a palha de aveia preta favoreceu as perdas de $\mathrm{N}$ para a atmosfera por volatilização de $\mathrm{NH}_{3}$ (Figura 2). Ao final do experimento (12,5 dias), a emissão dessa forma gasosa de $\mathrm{N}$, provocado pela aplicação dos dejetos, foi de $26,5 \mathrm{~kg}$ de $\mathrm{N}-\mathrm{NH}_{3} \mathrm{ha}^{-1}$ no solo sem resíduos culturais (Figura 2b), o que corresponde a 22,3\% do $\mathrm{N}$ amoniacal aplicado com os dejetos (Tabela 1) e de $17,5 \mathrm{~kg}$ de $\mathrm{N}-\mathrm{NH}_{3} \mathrm{ha}^{-1}$ sobre os resíduos culturais de aveia (14,7\% do $\mathrm{N}$ amoniacal aplicado). Essa redução em $9,0 \mathrm{~kg}$ de $\mathrm{N}_{-\mathrm{NH}_{3}}$ ha $^{-1}$ (34\%) na volatilização de $\mathrm{NH}_{3}$ evidencia a importância da presença de resíduos culturais quando os dejetos são aplicados na superfície do solo.

A emissão acumulada de $\mathrm{NH}_{3}$ (Figura 2b) mostra que aproximadamente $80 \%$ das perdas de $\mathrm{N}$ por volatilização ocorreu nas primeiras 22 horas após a aplicação dos dejetos, reduzindo rapidamente com o tempo. Essa cinética observada nas emissões de $\mathrm{NH}_{3}$ pode ser atribuída ao teor elevado de $\mathrm{N}$ amoniacal dos DLS (Tabela 1), que constitui um dos principais fatores que influencia na volatilização de $\mathrm{NH}_{3}$ (SOMMER \& HUTCHINGS, 2001). Além do $\mathrm{N}$ amoniacal, o pH inicial elevado dos dejetos é outro fator que contribui para tais emissões (MKHABELA et al., 2009). Corroborando com esses resultados, MEADE et al. (2011) constataram que 95\% das

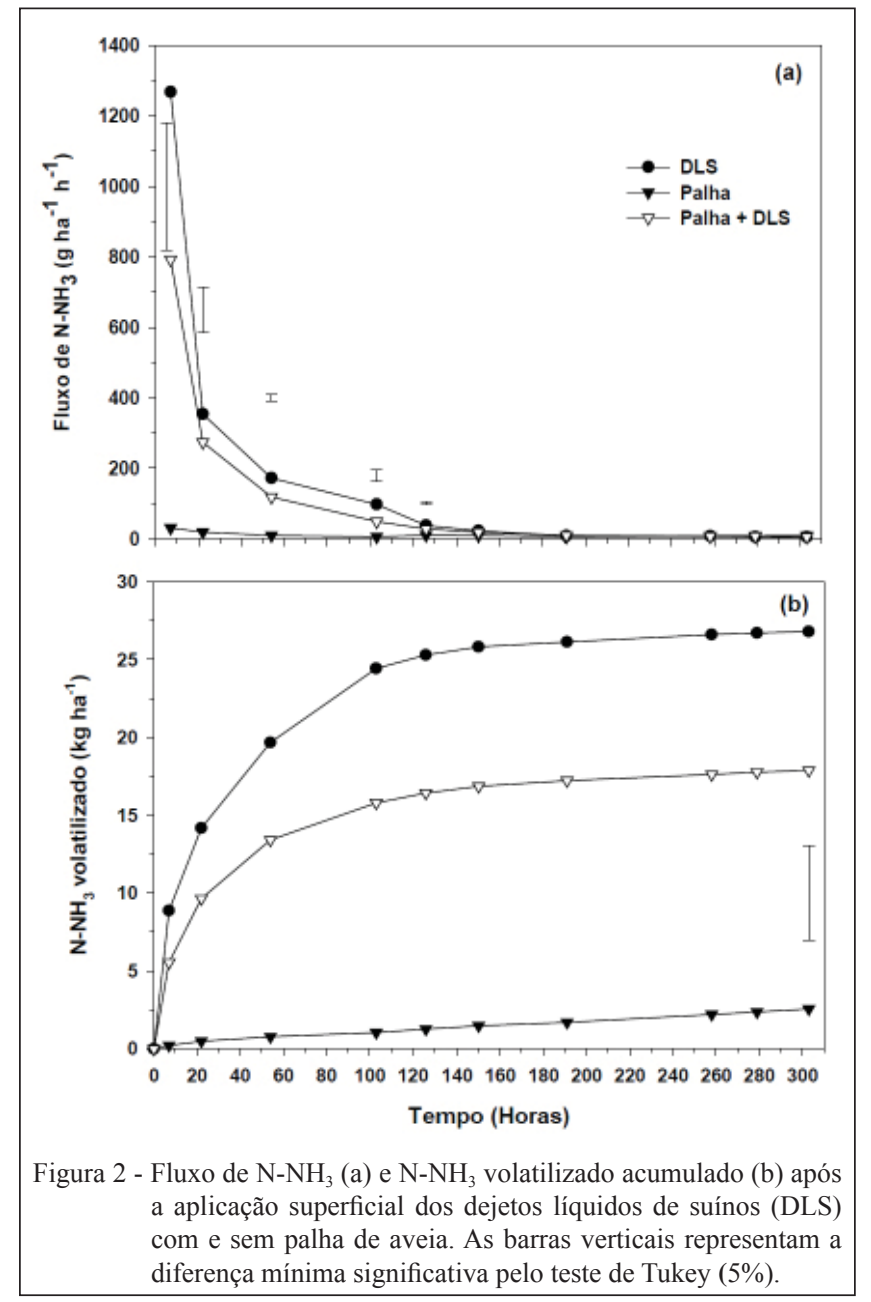

Ciência Rural, v.43, n.9, set, 2013. 
emissões de $\mathrm{NH}_{3}$ ocorreram nas primeiras 24 horas após a aplicação dos dejetos.

Comparando os tratamentos com aplicação de DLS, sem resíduos culturais e no solo com palha de aveia na superfície, observa-se que a presença da palha reduziu a emissão acumulada de $\mathrm{N}_{-} \mathrm{NH}_{3}$ em $34 \%$ (Figura $2 \mathrm{~b}$ ) ao final do experimento. Esse efeito da palha de aveia na redução das emissões de $\mathrm{NH}_{3}$ pode ser atribuído a diversos fatores. Ela atua como anteparo, reduzindo a ação do vento e a incidência do sol. De acordo com SOMMER et al. (2003), a redução da temperatura do ar e da velocidade do vento facilita a taxa de infiltração no solo do $\mathrm{N}$ amoniacal contido nos DLS, o que reduz a volatilização de $\mathrm{NH}_{3}$. Além disso, a aplicação de dejetos no solo sem resíduos culturais pode ocasionar a obstrução dos poros do solo, através da fração orgânica particulada presente nos dejetos, aumentando a concentração de $\mathrm{N}$ amoniacal na superfície do solo e, com isso, favorecer a volatilização de $\mathrm{NH}_{3}$. A palha também pode absorver parte da fração líquida dos dejetos, a qual é rica em $\mathrm{N}$ amoniacal. Com isso, menor quantidade de $\mathrm{N}$ amoniacal dos dejetos fica exposta à volatilização nas primeiras horas após a aplicação deles no solo.

A imobilização do $\mathrm{N}$ amoniacal dos DLS pelos microrganismos do solo, atuantes na decomposição da palha pode ser outro fator responsável pela menor emissão de $\mathrm{NH}_{3}$ no tratamento com aplicação dos dejetos sobre a palha. Em um experimento de campo, CHANTIGNY et al. (2001) verificaram que a adição de DLS sobre palha de cevada, com a incorporação de ambos, resultou em rápida imobilização de $\mathrm{N}$, atingindo, ao final dos primeiros 3 dias, $36 \%$ do $\mathrm{N}-\mathrm{NH}_{4}$ adicionado com os DLS. Embora, no presente trabalho, a palha de aveia tenha permanecido na superfície do solo, a água de irrigação e das chuvas pode ter promovido a liberação de $\mathrm{C}$ solúvel e outros constituintes dos resíduos culturais, favorecendo a população microbiana heterotrófica do solo, conforme destacado por AITA et al. (2007).

Apesar de os resultados do presente trabalho mostrar que a presença de resíduos culturais da aveia na superfície do solo reduziu significativamente a perda de $\mathrm{N}$ dos DLS por volatilização de $\mathrm{NH}_{3}$, considera-se importante que, em trabalhos futuros, sejam avaliadas as causas determinantes dessa redução, procurando variar a qualidade e a quantidade de resíduos culturais.

\section{CONCLUSÃO}

Quando os dejetos líquidos de suínos são aplicados sobre a palha de aveia, ocorre redução na perda de $\mathrm{N}$ por volatilização de $\mathrm{NH}_{3}$ em relação a sua aplicação no solo sem palha. No entanto, essa prática aumenta a emissão de $\mathrm{N}_{2} \mathrm{O}$.

\section{REFERENCIAS}

ABIPECS (ASSOCIAÇÃO BRASILEIRA DA INDÚSTRIA PRODUTORA E EXPORTADORA DE CARNE SUÍNA). Disponível em: <http://www.abipecs.org.br/pt/estatisticas/mundial/ exportacao.html>. Acesso em: 20 set. 2012.

AITA, C. et al. Nitrificação do nitrogênio amoniacal de dejetos líquidos de suínos em solo sob sistema de plantio direto. Pesquisa Agropecuária Brasileira, v.42, n.1, p.95-102, 2007. Disponível em: <www.scielo.br/pdf/pab/v42n1/13.pd>. Acesso em: 13 set. 2012. doi: http://dx.doi.org/10.1590/S0100-204X2007000100013.

BASSO et al. Perdas de nitrogênio de dejeto líquido de suínos por volatilização de amônia. Ciência Rural, v.34, n.6, p.17731778, 2004. Disponível em: <http://www.scielo.br/pdf/cr/v34n6/ a16v34n6.pdf>. Acesso em: 20 nov. 2012.

CHANTIGNY, M.H. et al. Short-term C in the cumulative $\mathrm{NH}_{3}-\mathrm{N}$ losses were not statistically and $\mathrm{N}$ dynamics in a soil amended with pig slurry and barley straw: a field experiment. Canadian Journal of Soil Science, v.81, n.2, p.131-137, 2001. Disponível em: <http:// pubs.aic.ca/doi/abs/10.4141/S00-046>. Acesso em: 13 set. 2012. doi: $10.4141 /$ S00-046.

GIACOMINI, S.J. et al. Emissão de óxido nitroso com a aplicação de dejetos líquidos de suínos em solo sob plantio direto. Pesquisa Agropecuária Brasileira, v.42, n.11, p.1653-1661, 2006. Disponível em: <http://www.scielo.br/pdf/pab/v41n11/a12v4111. pdf>. Acesso em: 10 set. 2012. doi: http://dx.doi.org/10.1590/ S0100-204X2006001100012.

HOEKSTRA, N.J. et al. Slurry ${ }^{15} \mathrm{NH}_{4}-\mathrm{N}$ recovery in herbage and soil: effects of application method and timing. Plant and Soil, v.330, n.1-2, p.357-368, 2010. Disponível em: <http://www. springerlink.com/content/5r7190j4t2771191/>. Acesso em: 15 set. 2012. doi: 0.1007/s11104-009-0210-z.

KUNZ, A. et al. Advanced swine manure treatment and utilization in Brazil. Bioresource Technology, v.100, n.22, p.5485-5489, 2009. Disponível em: <http://www.sciencedirect.com/science/ article/pii/S0960852408009218>. Acesso em: 12 set. 2012. doi: http://dx.doi.org/10.1016/j.biortech.2008.10.039.

MEADE, G. et al. Ammonia and nitrous oxide emissions following land application of high and low nitrogen pig manures to winter wheat at three growth stages. Agriculture, Ecosystems and Environment, v.140, n.1-2, p.208-217, 2011. Disponível em: <http://www.sciencedirect.com/science/article/ pii/S0167880910003221\#>. Acesso em: 21 set. 2012. doi: http:// dx.doi.org/10.1016/j.agee.2010.12.007.

MEIJIDE, A. et al. Nitrogen oxide emissions from an irrigated maize crop amended with treated pig slurries and composts in a Mediterranean climate. Agriculture, Ecosystems and Environment, v.121, n.4, p.383-394, 2007. Disponível em: <http://www.sciencedirect.com/ science/article/pii/S016788090600452X>. Acesso em: 18 set. 2012. doi: http://dx.doi.org/10.1016/j.agee.2006.11.020.

MKHABELA, M.S et al. The impact of management practices and meteorological conditions on ammonia and nitrous oxide

Ciência Rural, v.43, n.9, set, 2013. 
emissions following application of hog slurry to forage Grass in Nova Scotia. Agriculture, Ecosystems and Environment, v.130, n.1-2, p.41-49, 2009. Disponível em: <http://www.sciencedirect. com/science/article/pii/S0167880908003022>. Acesso em: 18 set. 2012. doi: http://dx.doi.org/10.1016/j.agee.2008.11.012.

NÔMMIK, H. The effect of pellet size on the ammonia loss from urea applied to forest soil. Plant and Soil, v.39, n.2, p.309-318, 1973. Disponível em: <http://www.springerlink.com/content/ u833m82hw2p271t1/>. Acesso em: 20 ago. 2012. doi: 10.1007/ BF00014798.

PERÄLÄ, P. et al. Influence of slurry and mineral fertilizer application techniques on $\mathrm{N}_{2} \mathrm{O}$ and $\mathrm{CH}_{4}$ fluxes from a barley Field in southern Finland. Agriculture, Ecosystems and Environment, v.117, n.1, p.71-78, 2006. Disponível em: <http:// www.sciencedirect.com/science/article/pii/S0167880906001289>. Acesso em: 15 ago. 2012. doi: http://dx.doi.org/10.1016/j. agee.2006.03.027.

PETERSEN, S.O.; SOMMER, S.G. Ammonia and nitrous oxide interactions: roles of manure organic matter management. Animal Feed Science and Technology, v.166-167, n.null, p.503-513, 2011. Disponível em: <http://www.journals.elsevierhealth.com/ periodicals/anifee/article/S03778401\%2811\%2900196-9/abstract>. Acesso em: 12 set. 2012. doi: 10.1016/j.anifeedsci.2011.04.077.
ROCHETTE, P.; BERTRAND, N. Soil-surface gas emissions. In: CARTER, M.; GREGORICH, E.G. (Eds.), Soil sampling and methods of analysis. Boca Raton, FL: CRC, 2008. p. 851-861.

SANTOS, H.G. dos et al. (Eds.). Sistema brasileiro de classificação de solos. 2.ed. Rio de Janeiro: Embrapa Solos, 2006. 306p. il. Inclui apêndices.

SOMMER, S.G. et al. Processes controlling ammonia emission from livestock slurry in the field. European Journal Agronomy, v.19, n.4, p.465-486, 2003. Disponível em: <http://www. sciencedirect.com/science/article/pii/S1161030103000376>. Acesso em: 15 out. 2012. doi: http://dx.doi.org/10.1016/S11610301(03)00037-6.

SOMMER, S.G.; HUTCHINGS, N.J. Ammonia emission from field applied manure and its reduction: invited paper. European Journal of Agronomy, v.15, n.1, p.1-15, 2001. Disponível em: <http://www.sciencedirect.com/science/article/pii/ S1161030101001125>. Acesso em: 15 out. 2012. doi: http://dx.doi. org/10.1016/S1161-0301(01)00112-5.

TEDESCO, M.J. et al. Análises de solo, plantas e outros materiais. 2.ed. Porto Alegre: UFRGS, 1995. 174p. (Boletim Técnico, 5). 